\title{
Taux de reproduction en fonction de la concentration en nourriture et de la température chez trois espèces du genre Brachionus (Rotifères)
}

\author{
R. Pourriot ${ }^{1}$ \\ C. Rougier ${ }^{2}$
}

Mots clés : reproduction, concentration algale, température, Rotifères, Brachionus.

L'influence des variations de la quantité de nourriture (de $5.10^{3}$ à $1.10^{7}$ cell.mL-1 de chlorelles) sur la dynamique de populations est étudiée à deux températures $\left(16 \mathrm{et} 24^{\circ} \mathrm{C}\right.$ ) chez trois espèces, Brachionus angularis, B. calyciflorus (représentée par deux clones) et $B$. plicatilis. En observant isolément une quinzaine de femelles, on évalue le taux net de reproduction, $\mathrm{R}_{0}$, le taux intrinsèque de croissance, $\mathrm{r}_{\mathrm{m}}$, la quantité minimale de nourriture pour une croissance nulle, $\mathrm{S}_{0}$, et la concentration algale, $\mathrm{Ks}$, nécessaire pour atteindre un taux de croissance maximale (ou la moitié). Les valeurs obtenues pour ces variables sont comparées entre elles et à celles de la littérature. La souche de $B$. plicatilis se singularise par des valeurs particulièrement bassés. Les auteurs relient cette particularité au métabolisme propre à cette espèce adaptée à de fortes variations de salinité et de nourriture.

Reproduction rates in relation to food concentration and temperature in three species of the genus Brachionus (Rotifera)

Keywords : reproduction, algal concentration, temperature, Rotifers, Brachionus.

The influence of various algal (Chlorella) concentrations (from $5.10^{3}$ to $1.10^{7}$ cells $\mathrm{mL}^{-1}$ ) on the population dynamic is studied at two temperatures $\left(16 \& 24^{\circ} \mathrm{C}\right.$ ) in three species, Brachionus angularis, B. calyciflorus (with two clones) and B. plicatilis. By observing about fifteen isolated females, the net reproduction rate, $R_{0}$, the intrinsic population growth rate, $r_{m}$, the threshold food level (for which $r_{m}=0$ ), $S_{0}$, and the algal concentration, Ks, supporting the maximum population growth rate (or one-half) are determined. The values of these variables are compared with each others and with the literature data. The low values observed in the $B$. plicatilis strain are conspicuous. This peculiarity is related to the metabolism of the species adapted to various salinity and food conditions.

\section{Introduction}

Il est couramment admis que les deux facteurs biotiques, prédation et compétition, concourrent à structurer les populations (Pourriot 1995). Toutefois, les effets de la prédation étaient jusqu'à présent mieux compris, en tous cas plus étudiés, que ceux de la compétition pour les ressources (Kerfoot \& Sih 1987, Lazzaro \& Lacroix 1995). Le rôle de la compétition sous ses différents aspects a été récemment revu par De Mott (1989) et, chez les Rotifères, par Rothhaupt (1990).

1. Université Paris 6, URA 1367, Laboratoire de Géologie Appliquée, B 123, 4 place Jussieu, F-75252 Paris Cédex 05

2. Université Montpellier II, UMR 5556, Laboratoire d'Hydrobiologie marine et continentale, Case 093, F-34095 Montpellier Cédex 5.
Ces dernières années, plusieurs auteurs ont contribué à clarifier les concepts théoriques invoqués dans l'interprétation des phénomènes de compétition pour les ressources nutritives et ont attiré l'attention sur une approche démographique tant chez les Rotifères (Stemberger \& Gilbert 1985, Rothhaupt 1990b) que chez les Cladocères (Gliwicz 1990, Duncan 1989). Parmi ces concepts figurent :

- le seuil minimal $\left(S_{O}\right)$ de concentration alimentaire pour une croissance nulle de la population $\left(r_{m}=0\right)$, c'est-à-dire le maintien de celle-ci à un état d'équilibre, et

- la concentration (Ks) nécessaire pour atteindre le taux maximal de croissance $\left(r_{\max }\right)$ ou sa moitié $\left(\mathrm{r}_{\max } / 2\right)$, considérée comme une expression de l'adaptation physiologique au niveau de ressource (Crowley 1975, in Stemberger \& Gilbert 1985).

La plupart de ces travaux ont mis en évidence la relation interspécifique existant entre le seuil minimal 
$S_{\mathrm{O}}$, et la taille du corps. En outre, Rothhaupt (1990a) a démontré l'existence d'une relation intraspécifique entre ce seuil et la taille des particules alimentaires.

Au cours d'expériences où la nature et la taille de la source nutritive n'ont pas varié, nous nous sommes efforcés de comparer le taux de reproduction et les variables $S_{O}$ et Ks chez trois espèces d'un même genre, herbivores polyphages, à deux températures.

\section{Matériel et méthodes}

Les animaux testés proviennent de quatre clones élevés en laboratoire et appartenant à trois espèces :

- Brachionus angularis S89, d'une femelle amictique récoltée dans la Seine en juin 1989;

- B. calyciflorus Cr79, d'une femelle isolée du plancton du lac de Créteil en mars 1979;

- B. calyciflorus A89, d'une femelle éclose en mai 1989 d'oeufs de durée provenant d'un lagunage d'Allemagne;

- B. plicatilis GS74, d'une femelle éclose en 1974 d'oeufs de durée présents dans la vase prélevée dans les Garcines Sud de Camargue en 1969.

Le milieu d'élevage est constitué pour les trois premiers d'une eau de source naturelle peu minéralisée (eau de Volvic) et pour le quatrième, d'eau de mer en provenance de la Manche et diluée au 1/3 (concentration moyenne des eaux saumâtres où se rencontre $B$. plicatilis). Tous sont alimentés de Chlorella pyrenoïdosa (Chlorophycée, Chlorococcalé), cultivée sur milieu synthétique (Pourriot 1980). Cette algue sphérique, de $3,5 \mu \mathrm{m}$ de diamètre, est située dans la gamme de taille préférentiellement consommée par les trois espèces, même si la taille maximale des particules ingérables (jusqu'à $20 \mu \mathrm{m}$ ) est largement supérieure pour les deux dernières, de taille semblable mais supérieure à B. angularis (Rothhaupt $1990 \mathrm{a}$, Vadstein et al. 1993).

Les concentrations algales utilisées s'échelonnent de $5.10^{3}$ à $10^{7}$ cellules par millilitre. Selon la formule préconisée par Rocha \& Duncan (1985), la teneur en carbone est de 3,15 pg C par cellule. En admettant une teneur en carbone égale à $50 \%$ de la masse sèche, celleci représente environ $28 \%$ du biovolume, coefficients de conversion acceptables selon Reynolds (1984).

Deux températures, $16^{\circ} \mathrm{C}$ et $24^{\circ} \mathrm{C}$, ont été expérimentées car les deux clones de $B$. calyciflorus présentent une relative sténothermie, la souche CR79 ne se reproduisant pas à haute température alors que la souche $\mathrm{A} 89$ se reproduit mal à basse température. Les clones sont couramment maintenus en enceinte obscure à la température de $16^{\circ} \mathrm{C}$, sauf $B$. calyciflorus A89 élévé à $24^{\circ} \mathrm{C}$. Les trois autres clones sont adaptés une semaine à cette température avant expérimentation.

Les masses corporelles des Rotifères sont calculées à partir des biovolumes établis par la formule de Ruttner-Kolisko (1977), $\mathrm{V}=0,12 \mathrm{~L}^{3}$, en admettant que la densité est égale à 1 et que la masse sèche (MS) représente $10 \%$ du biovolume (Bottrell et al. 1976).

\section{Protocole expérimental}

Des femelles ovigères sont extraites des élevages et isolées dans des cellules contenant $1 \mathrm{~mL}$ de milieu avec une quantité d'algues correspondant à la concentration testée. Le lendemain, des nouveaux-nés sont isolés dans les mêmes conditions. Seules, les femelles amictiques sont conservées et suivies jusqu'à leur mort. Le milieu et les algues nutritives sont changés quotidiennement et les jeunes femelles écloses comptées et enlevées. En admettant un taux de filtration maximum de

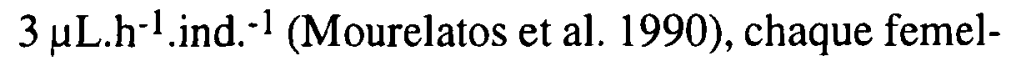
le ovigère consomme journellement environ $7 \%$ de la concentration algale initiale, ce qui ne modifie que faiblement la quantité d'algues disponible.

Cette procédure permet d'évaluer le nombre moyen de jeunes produits par une femelle durant sa vie et sa variance, peu différent du taux net de reproduction, $\mathrm{R}_{0}$ $\left(=\Sigma l_{x} \mathrm{~m}_{\mathrm{x}}\right.$, où $\mathrm{l}_{\mathrm{x}}$ est la probabilité de survie à l'âge $\mathrm{x}$ et $\mathrm{m}_{\mathrm{x}}$, la fécondité à l'âge $\mathrm{x}$ ). A partir de ce taux net, il est possible d'évaluer le taux intrinsèque d'accroissement, $\mathrm{r}_{\mathrm{m}}$, pour chaque concentration nutritive par la méthode de Birch (1948).

Le seuil minimal de nourriture (pour $r_{m}=0$ ) est déterminé graphiquement en traçant une ligne entre les valeurs positives et négatives de $r_{m}$ proches de zéro. En absence de valeurs négatives, la ligne est extrapolée à partir des valeurs les plus basses (Stemberger \& Gilbert 1985).

\section{Résultats}

La fertilité quotidienne moyenne d'une femelle amictique varie selon un mode classique chez $B$. angularis et $B$. calyciflorus où elle présente un maximum dans les premiers jours de la vie de la femelle mère aux concentrations algales élevées (Fig. 1). Chez B. plicatilis, ce pic se situe vers la moitié de la période de fécondité. Dans tous les cas, ce maximum s'atténue puis disparaît à mesure que les ressources alimentaires disponibles diminuent.

Le nombre maximum de jeunes produits en moyenne par une femelle durant sa vie est d'environ $27 \mathrm{chez}$ $B$. plicatilis et 19 chez $B$. calyciflorus mais seulement de 9 chez B. angularis (Tableau 1). Le taux net de reproduction, $R_{0}$, croît avec la concentration algale selon 

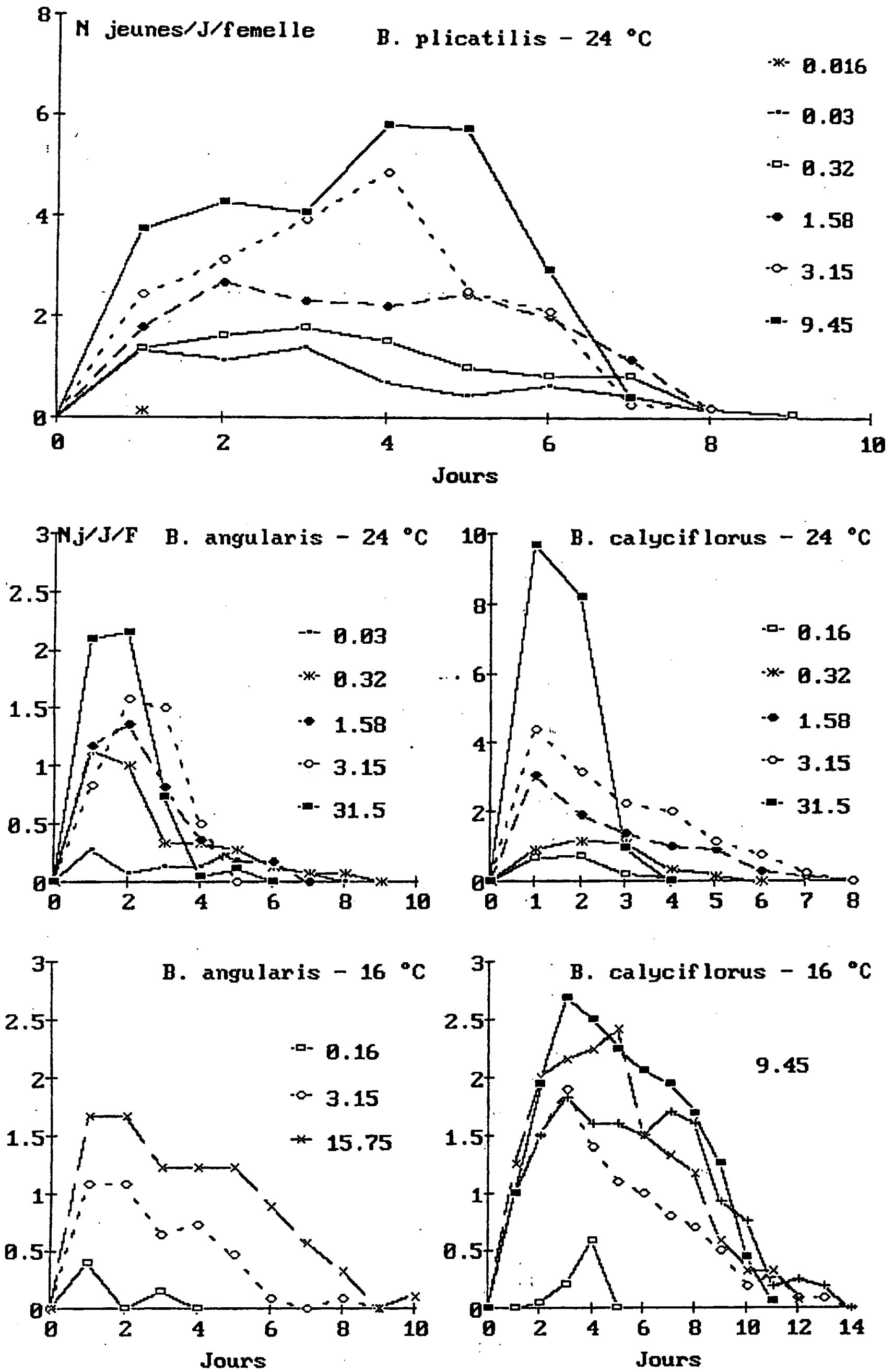

Fig. 1. Variations de la fécondité journalière avec l'âge de la femelle à différentes concentrations algales, exprimées en mg $\mathrm{C} / \mathrm{L}$, et à deux températures chez trois espèces de Brachionus.

Fig. 1. Variations of the daily fecundity with the age of the female at various algal concentrations, expressed in $\mathrm{mg}$ $\mathrm{C} / \mathrm{L}$, and at two temperatures in three species of the genus Brachionus. 
Tableau 1. Nombre de jeunes produits par une femelle durant sa vie $\left(R_{0}\right)$ et taux intrinsèque de croissance, $r_{m}$ (femelle.femelle ${ }^{-1}$.jour ${ }^{-1}$ ), à différentes concentrations de nourriture et deux températures chez trois espèces planctoniques. $\mathrm{N}:$ nombre de femelles; $\mathrm{L}: \mathrm{lon}$ gueur en $\mu \mathrm{m}$, le nombre d'individus mesurés est indiqué entre parenthèses.

Table 1. Number of youngs produced per female during her lifetime $\left(R_{0}\right)$, intrinsic rate of population growth, $r_{m}\left(\right.$ female.female $e^{-1}$.day $\left.{ }^{-1}\right)$, at various concentrations of food and at two temperatures in three planktonic species. $N$ : number of females; $L:$ body length in $\mu \mathrm{m}$ (the number of measures is noted in brackets.

\begin{tabular}{|c|c|c|c|c|c|}
\hline Espèce & $\mathrm{T}^{\circ} \mathrm{C}$ & $\operatorname{cel} 1 . \mathrm{mL}^{-1}$ & $\mathbf{N}$ & $\mathrm{k}_{0} \pm \mathrm{e} \cdot \mathrm{t}$ & $I_{m}$ \\
\hline $\begin{array}{c}\text { B. } \text { plicatilis } \\
\text { (GS } 74) \\
\mathrm{L}=242,9 \pm 10,1 \\
(29)\end{array}$ & 24 & $\begin{array}{l}5.10^{3} \\
1.10^{4} \\
5.10^{4} \\
5.10^{5} \\
1.10^{6} \\
3.10^{6}\end{array}$ & $\begin{array}{l}21 \\
16 \\
19 \\
13 \\
15 \\
15\end{array}$ & $\begin{array}{r}0,14 \\
6,1 \pm 2,2 \\
9,7 \pm 2,5 \\
14,8 \pm 4,1 \\
19,5 \pm 6,1 \\
26,9 \pm 3,8\end{array}$ & $\begin{array}{r}-0,500 \\
0,385 \\
0,500 \\
0,539 \\
0,617 \\
0,707\end{array}$ \\
\hline $\begin{array}{l}\text { B. calyciflorus } \\
(A 89) \\
L=199,7 \pm 11 \\
(20)\end{array}$ & 24 & $\begin{array}{l}1.10^{4} \\
5.10^{4} \\
1.10^{5} \\
5.10^{5} \\
1.10^{6} \\
1.10^{7}\end{array}$ & $\begin{array}{l}15 \\
17 \\
18 \\
13 \\
13 \\
20\end{array}$ & $\begin{array}{r}0 \\
1,6 \pm 0,8 \\
3,7 \pm 1,4 \\
8,8 \pm 3,8 \\
13,9 \pm 4,6 \\
18,8 \pm 3,1\end{array}$ & $\begin{array}{c}0 \\
0,123 \\
0,311 \\
0,537 \\
0,649 \\
0,862\end{array}$ \\
\hline $\begin{array}{l}\text { B. Calyciflorus } \\
(\text { Cr } 79) \\
I=230,9 \pm 15,2 \\
(15)\end{array}$ & 16 & $\begin{array}{l}5.10^{4} \\
1.10^{6} \\
3.10^{6} \\
5.10^{6} \\
1.10^{7}\end{array}$ & $\begin{array}{l}19 \\
10 \\
12 \\
12 \\
16\end{array}$ & $\begin{array}{r}0,9 \pm 0,3 \\
10,6 \pm 2,5 \\
14,5 \pm 4,0 \\
15,4 \pm 4,8 \\
17,8 \pm 3,3\end{array}$ & $\begin{array}{r}-0,025 \\
0,354 \\
0,371 \\
0,407 \\
0,412\end{array}$ \\
\hline $\begin{array}{l}\text { B. angularis } \\
(\mathrm{Cr} 89) \\
\mathrm{L}=127,8 \pm 5,9 \\
(20)\end{array}$ & 24 & $\begin{array}{l}1.10^{4} \\
1.10^{5} \\
5.10^{5} \\
1.10^{6} \\
1.10^{7}\end{array}$ & $\begin{array}{l}15 \\
15 \\
11 \\
12 \\
20\end{array}$ & $\begin{array}{l}1,1 \pm 1,4 \\
3,3 \pm 1,7 \\
4,1 \pm 1,8 \\
4,4 \pm 1,5 \\
5,2 \pm 2,1\end{array}$ & $\begin{array}{l}0,014 \\
0,284 \\
0,340 \\
0,350 \\
0,446\end{array}$ \\
\hline B. angularis & 16 & $\begin{array}{l}5.10^{4} \\
1.10^{6} \\
5.10^{6}\end{array}$ & $\begin{array}{r}13 \\
11 \\
9\end{array}$ & $\begin{array}{l}0,5 \pm 0,8 \\
4,2 \pm 1,9 \\
8,8 \pm 2,9\end{array}$ & $\begin{array}{r}0,046 \\
0,219 \\
0,284\end{array}$ \\
\hline
\end{tabular}

une fonction croissante monotone avec une asymptote, telle une fonction puissance avec l'exposant $b<1$ par exemple (Fig. 2). Chacune des trois espèces présente une variation caractéristique peu influencée par la température. La corrélation entre $\mathrm{R}_{0}$ et le logarithme de la concentration nutritive est très significative $(p>0.01)$ et la régression, linéaire (Tableau 2). Le taux intrinsèque de croissance, $r_{m}$, calculé à partir des données précédentes, montre des caractèristiques similaires (Fig. 3).

Le taux de mortalité des embryons et des juvéniles est faible chez $B$. plicatilis $(<5 \%)$ de même que chez $B$. calyciflorus où il a tendance à augmenter aux basses concentrations nutritives mais reste élevé à toutes les concentrations chez B. angularis (Tableau 3).

Les valeurs du seuil de ressources, $S_{0}$, pour une croissance nulle et Ks pour un taux de croissance maximal, observées à chaque température pour les quatre clones varient respectivement de 0,02 à 0,19 et de 0,03 à $1,0 \mu \mathrm{g} \mathrm{C} \mathrm{mL}^{-1}$ (Tableau 4). $B$. angularis et $B$. plicatilis montrent les valeurs les plus faibles et $B$. $c a$ lyciflorus, les plus fortes quelle que soit la température.

\section{Discussion}

Des valeurs de $R_{0}$ et $r_{m}$ proches des nôtres sont couramment obtenues pour $B$. plicatilis et pour $B$. calyci- 


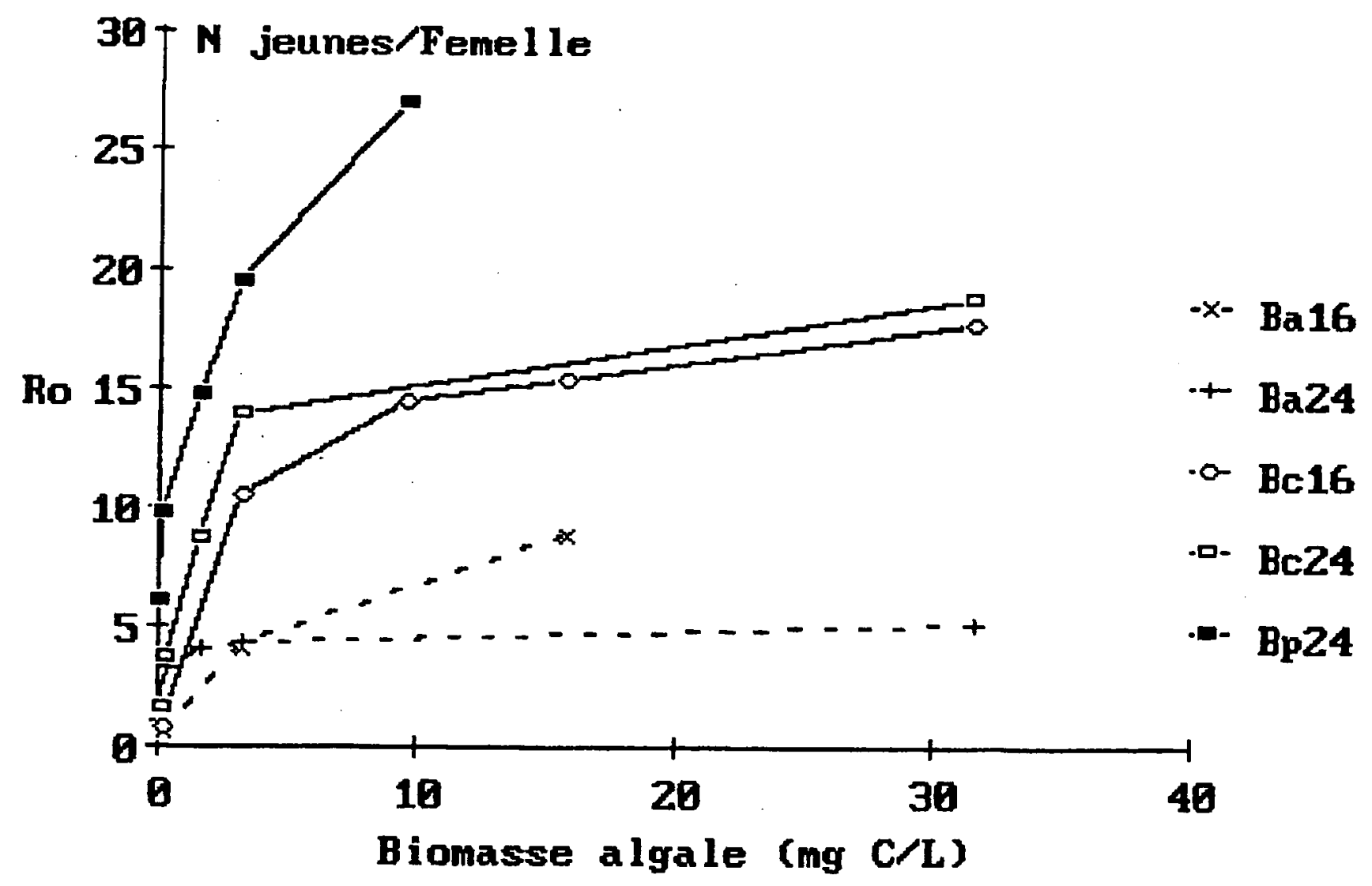

Fig. 2. Variations du $\mathrm{R}_{0}$ en fonction de la biomasse algale chez les trois espèces à deux températures. $\mathrm{Ba}: \mathrm{B}$. angularis, Bc : B. calyciflorus, $\mathrm{Bp}:$ B. plicatilis, $16=16^{\circ} \mathrm{C}, 24=24^{\circ} \mathrm{C}$.

Fig. 2. Variations of $\mathrm{R}_{0}$ with the algal biomass in three species at two temperatures.

Tableau 2. Valeurs des paramètres a,b, dans l'équation de régression $\mathrm{R}_{0}=\mathrm{a}+\mathrm{b} .1 \mathrm{nC}$, et du coefficient de détermination, $\mathrm{r}^{2}, \mathrm{C}$, concentration en nourriture, en $\mathrm{mg} \mathrm{C} \mathrm{L} \mathrm{L}^{-1}$, d'après le tableau 1.

Table 2. Values of parameters $a, b$, in the regression equation $R_{0}=a+b \cdot \operatorname{lnC}$, and of the determination coefficient, $r^{2}$. $C$, food concentration in $\mathrm{mg} \mathrm{C} \mathrm{L}^{-1}$, following the data in table 1.

\begin{tabular}{lcccccc}
\hline Espèce & $\mathrm{T}^{\circ} \mathrm{C}$ & \multicolumn{1}{c}{ a } & E.S. & b & E.S. & $\mathrm{r}^{2}$ \\
\hline B. plicatilis & 24 & 17.61 & 1.14 & 3.60 & 0.42 & 0.948 \\
B. calyciflorus & 24 & 9.63 & 0.74 & 2.97 & 0.32 & 0.955 \\
B. Calyciflorus & 16 & 8.08 & 0.17 & 3.21 & 0.08 & 0.998 \\
B. angularis & 24 & 3.79 & 0.22 & 0.58 & 0.09 & 0.929 \\
B. angularis & 16 & n.s. & & & & \\
\hline
\end{tabular}

florus dans des conditions peu différentes, voire identiques (Tableau 5).

Le taux net de reproduction ainsi que le taux intrinsèque de croissance, qui lui est lié, s'accroissent rapidement avec l'augmentation des ressources tant que celles-ci sont limitantes $\left(<6 \mathrm{mg} \mathrm{C} \mathrm{L}^{-1}\right)$. Cette réponse est similaire à celle obtenue par d'autres auteurs expérimentant avec une gamme identique de concentra- tions algales chez les mêmes espèces de Rotifères (Hiramaya et al. 1973, Hallbach \& Hallbach-Keup 1974, Schmid-Araya 1991). Aux concentrations algales supérieures, ces auteurs observent un optimum du nombre de descendants par femelle suivi d'une décroissance qui n'apparaît pas dans nos expériences où la relation entre $R_{0}$ et la concentration algale est de type logarithmique (Fig. 2, Tableau 2). 


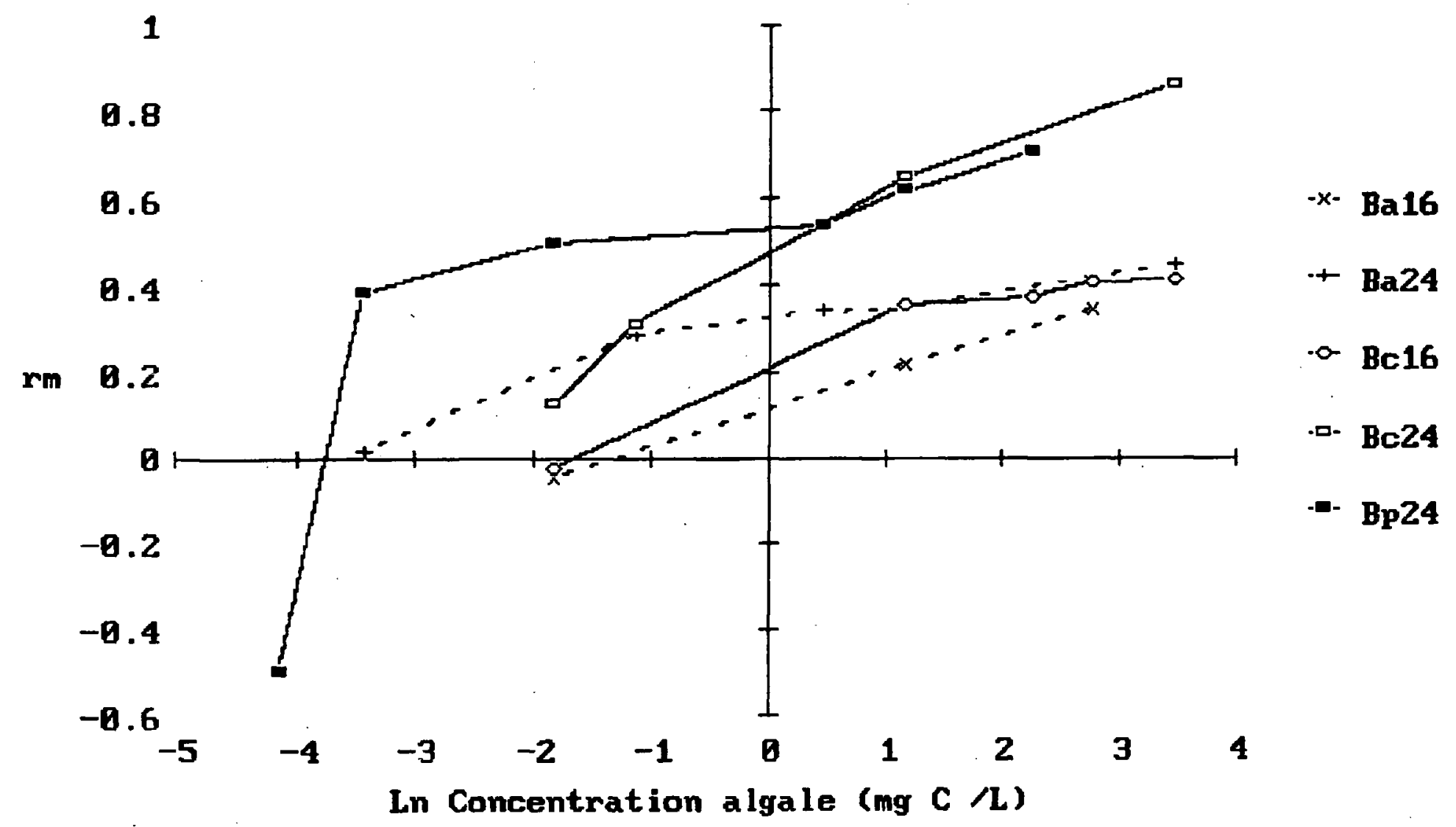

Fig. 3. Variations de $\mathrm{r}_{\mathrm{m}}$ en fonction de la biomasse algale chez les trois espèces à deux températures. $\mathrm{Ba}: B$. angularis, $\mathrm{Bc}: B$. calyciflorus, $\mathrm{Bp}: B$. plicatilis, $16=16^{\circ} \mathrm{C}, 24=24^{\circ} \mathrm{C}$.

Fig. 3. Variations of $r_{m}$ with the algal biomass in three species at two temperatures.

Tableau 3. Taux de mortalité, den $\%$ de $\mathrm{R}_{0}$, des embryons et des juvéniles de $B$. angularis et de $B$. calyciflorus à $24^{\circ} \mathrm{C}$ et à différentes concentrations en nourriture.

Table 3. Death rate of embryos and youngs, $d$ in $\%$ of $\mathrm{R}_{0}$, in B. angularis and B. calyciflorus at $24^{\circ} \mathrm{C}$ and at various food concentrations.

\begin{tabular}{|c|c|c|c|}
\hline Espèce & $\mathrm{N}$ cells $\mathrm{mL}^{-1}$ & $\mathrm{~d}$ & \\
\hline $\begin{array}{l}\text { B. calyciflorus } \\
(\text { A 89) }\end{array}$ & $\begin{array}{l}1.10^{4} \\
5.10^{4} \\
1.10^{5} \\
5.10^{5} \\
1.10^{6} \\
1.10^{7}\end{array}$ & $\begin{array}{r}- \\
- \\
9 \\
4 \\
<1\end{array}$ & $\begin{array}{l}\left(R_{0}=0\right) \\
\left(R_{0}=0\right)\end{array}$ \\
\hline 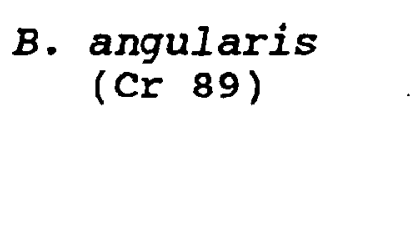 & $\begin{array}{l}1.10^{4} \\
1.10^{5} \\
5.10^{5} \\
1.10^{6} \\
1.10^{7}\end{array}$ & $\begin{array}{l}31 \\
26 \\
27 \\
34 \\
30\end{array}$ & \\
\hline
\end{tabular}


Tableau 4. Concentrations en aliment, Ks et $S_{0}$, en $\mu \mathrm{g} \mathrm{C} \mathrm{mL}-1$, pour deux valeurs du taux de croissance $\left(r_{\max } / 2\right.$ et $\left.r_{m}=0\right)$ évaluées chez les trois espèces. Grap. : valeurs déduites de la figure 3; calc. : valeurs calculées d'après les équations de Stemberger \& Gilbert (1985) : $\mathrm{S}_{0}=1,824 \mathrm{MS}^{0.832} ; \mathrm{Ks}=14,149 \mathrm{MS}^{1.223}$ (MS = masse sèche du corps).

Table 4. Food concentrations, $\mathrm{Ks}$ and $\mathrm{S}_{0}$, en $\mu \mathrm{g} \mathrm{C} \mathrm{mL}-1$, at two values of the growth rate $\left(r_{\max } / 2\right.$ et $\left.r_{\mathrm{m}}=0\right)$ estimated in the three species. Grap. : values infered from Fig. 3; calc. : values calculated from the equations of Stemberger \& Gilbert $(1985): \dot{S}_{0}=1,824$ $\mathrm{DM}^{0.832} ; \mathrm{Ks}=14,149 \mathrm{DM}^{1.223}$

\begin{tabular}{lcccccc}
\hline espèce & ${ }^{\circ}{ }^{\circ} \mathrm{C}$ & $\mathrm{r}_{\max } / 2$ & \multicolumn{2}{c}{$\mathrm{S}_{0}$} & \multicolumn{2}{c}{ Ks } \\
& & & grap. & calc. & \multicolumn{2}{c}{ grap. calc. } \\
\hline B. plicatilis GS74 & 24 & 0,353 & 0,02 & 0,21 & 0,03 & 0,81 \\
B. calyciflorus A89 & 24 & 0,431 & 0,10 & 0,135 & 0,67 & 0,43 \\
B. calyciflorus Cr79 & 16 & 0,206 & 0,19 & 0,185 & 1,0 & 0,70 \\
B. angularis Cr89 & 24 & 0,223 & 0,03 & 0,04 & 0,17 & 0,08 \\
\hline
\end{tabular}

Tableau 5. Données démographiques de deux espèces du genre Brachionus selon différentes sources. ESD : diamètre équivalent sphérique; $\mathrm{R}_{0}$ est exprimé en nombre d'œufs par femelle et $\mathrm{S}_{0}$, en $\mu \mathrm{g} \mathrm{MS} \mathrm{mL} \mathrm{m}^{-1}$; le tiret correspond à une absence de données.

Table 5. Demographic data on two species of Brachionus from various authors. ESD : sphaeric equivalent diameter ; $R_{0}$ is expressed in number of eggs per female and $S_{0}$, in $\mu \mathrm{g} \mathrm{DM} \mathrm{mL}^{-1}$; a dash = no data.

\begin{tabular}{|c|c|c|c|c|c|c|c|}
\hline $\mathrm{T}^{\circ} \mathrm{C}$ & $\mathrm{S} \%$ & Nourriture & ESD & $\mathbf{R}_{0}$ & $r_{\max }$ & $\mathrm{S}_{0}$ & référence \\
\hline \multicolumn{8}{|c|}{ B. calyciflorus } \\
\hline 1.9 & - & Cryptomonas & 5,9 & - & 0,82 & 0,38 & Stemb. \& Gilbert (1985) \\
\hline 20 & - & Monoraphidium & 3,5 & - & 0,785 & 0,47 & Rotthaupt (1990b) \\
\hline 24 & - & Chlorella & 3,5 & 18,8 & 0,86 & 0,21 & présent travail \\
\hline 16 & - & Chlorella & $3 ; 5$ & 17,8 & 0,41 & 0,38 & présent travail \\
\hline \multicolumn{8}{|c|}{ B. plicatilis } \\
\hline 20 & 17 & Dunaliella & 8,9 & 23 & 0,94 & - & Ruttner-Kolisko (1972) \\
\hline 22 & 23 & Chlorella & 1,34 & 20,1 & 0,71 & - & Hiramaya et al. (1973) \\
\hline 22 & 23 & Chlorella & 1,34 & 20 & 0,88 & - & Hiramaya et al. (1979) \\
\hline - & $\therefore$ & - & - & - & - & 0,40 & Stemb. \& Gilbert (1987) \\
\hline 25 & 15 & Dunaliella & 8,9 & 27,5 & - & 0,57 & Snell \& Boyer (1988) \\
\hline 20 & 16,5 & Brachiomonas & 9,7 & 19,6 & - & - & Schmid-Araya (199i) \\
\hline 24 & 12 & Chlorella & 3,5 & 26,9 & 0,71 & 0,06 & présent travail \\
\hline
\end{tabular}

L'influence négative des densités algales élevées peut être due à une toxicité des métabolites algaux (Hallbach \& Hallbach-Keup 1974) mais aussi à un transit intestinal trop rapide entraînant une assimilation partielle des aliments ou à une résistance des parois cellulaires à l'action des enzymes gastriques.

Un effet toxique ne semble pas intervenir dans cette expérimentation où le taux de mortalité des jeunes ne varie pas avec la concentration algale.

En revanche, une mauvaise digestibilité des chlorelles peut être suggérée pour $B$. angularis dont la faible fécondité s'accompagne d'une mortalité élevée et peu variable des jeunes (de 26 à $34 \%$ à $24^{\circ} \mathrm{C}$ à toutes les concentrations) alors que les deux autres espèces ont une fécondité supérieure et une mortalité juvénile faible et croissant légèrement avec la dilution de l'aliment (de 1 à $8 \%$ ). A $16^{\circ} \mathrm{C}$, la forte mortalité et le faible nombre de données ne permet pas de s'assurer d'un taux de croissance maximum.

Un taux net de reproduction $\left(\mathrm{R}_{0}=3,61\right)$ et un taux de croissance $\left(r_{m}=0,35\right)$ faibles accompagnés d'une mortalité élevée sont également observés par Walz 
(1987) pour $B$. angularis $\left(20^{\circ} \mathrm{C}\right.$, nourriture $=$ Stichococcus bacillaris). Ces caractères indiquent plus probablement une qualité de la source alimentaire médiocre pour cette espèce qu'une véritable stratégie de reproduction.

B. calyciflorus présente des valeurs de $\mathrm{S}_{0}$ et de $\mathrm{Ks}$ supérieures à celles de $B$. angularis et en accord avec celles calculées d'après l'équation établie par Stemberger \& Gilbert (1985 : Tableau 4). Au delà de la relation avec la taille, cette différence semble en rapport avec l'écologie de ces deux espèces, la première colonisant des milieux nettement plus eutrophes (hypereutrophes) que la seconde (Pourriot 1965). B. angularis montre des caractères de stratège «K», plus compétitive à basse concentration en nourriture mais moins en présence de ressources non limitées, par rapport à $B$. calyciflorus ayant une stratÉgie de type «r». La contradiction avec les observations de Walz (1987), qui considère $B$. angularis comme un stratège «r», n'est peut-être qu'apparente. En effet, cet auteur la compare à $K$. cochlearis et rien n'interdit l'existence d'un gradient $\mathrm{r}-\mathrm{K}$ allant de $B$. calyciflorus à $B$. angularis puis à $K$. cochlearis.

En ce qui concerne le seuil, $S_{0}$, les valeurs observées dans ce travail pour $B$. calyciflorus sont semblables à celles données par d'autres auteurs mais ce n'est pas le cas de celles obtenues pour $B$. plicatilis. Ainsi, chez $B$. calyciflorus, Rothhaupt (1990b) situe à $0,236 \mu \mathrm{g} \mathrm{C}$ $\mathrm{mL}^{-1}$ (soit $0,47 \mu \mathrm{g} \mathrm{MS} \mathrm{mL}^{-1}$ ) la valeur de $\mathrm{S}_{0}$ pour une algue de diamètre sphérique équivalent $\left(\right.$ à $20^{\circ} \mathrm{C}$; Tableau 5). La valeur $(0,38)$ fournie par Stemberger \& Gilbert (1985) diffère peu de celle de Rotthaupt quoique le rapport de la masse sèche au biovolume, estimé à 0,69 pour les Cryptomonadines utilisées comme source nutritive, paraisse bien élevé.

Il en va tout autrement chez B. plicatilis où Stemberger \& Gilbert. (1987) et Snell \& Boyer (1988) évaluent

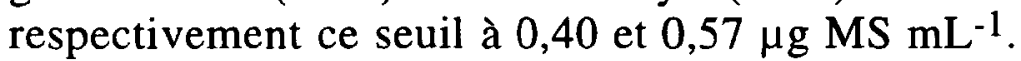
Les différences observées avec nos propres valeurs $\left(0,06 \mu \mathrm{g} \mathrm{MS} \mathrm{mL}^{-1}\right)$ atteignent un ordre de grandeur, ce qui est inhabituel.

Stemberger \& Gilbert $(1985,1987)$ ont montré que le logarithme de $S_{0}$ et celui de Ks étaient positivement et significativement corrélé au logarithme de la masse corporelle. En appliquant les équations données par ces auteurs à la masse individuelle des espèces testées, on obtient des valeurs de $S_{0}$ et Ks comparables aux valeurs lues graphiquement chez $B$. calyciflorus et $B$. angularis (quoique la valeur de Ks soit 3 fois supérieure chez cette dernière) mais d'un ordre de grandeur inférieur (soit environ 10 fois moins) chez B. plicatilis (Tableau 4).
Un écart de cet ordre ne paraît pas pouvoir être expliqué par la variabilité interspécifique ni par une différence de masse corporelle, ni par les différences entre les conditions expérimentales (de température en particulier) ou méthodologiques (calcul de la masse corporelle ou coefficients de conversion utilisés). Cette variation intraspécifique paraît propre à $B$. plicatilis représentée dans les milieux saumâtres par des populations isolées, de taille variable (Snell \& Hawkinson 1983, Snell \& Carrillo 1984). Ce caractère reflète vraisemblablement son aptitude à coloniser des eaux de statut trophique variable (Arfi et al. 1987, Gaudy et al. 1995), à supposer toutefois qu'il s'agisse d'une seule espèce avec une forte variation interclonale et non d'un complexe d'espèces (cf. Segers 1995).

Stemberger \& Gilbert (1987) suggèrent que les coûts énergétiques liés à la nage expliquent la relation taille du corps- $S_{0}$. Chez cette espèce d'eau saumâtre, la vitesse de nage varie vraisemblablement avec la viscosité fonction de la salinité de l'eau. La diminution de la consommation d'oxygène observée aux salinités élevées (in Pejler 1995) est l'indice d'une réduction du métabolisme. En outre, la vitesse de nage détermine les taux de rencontre de la nourriture (Stemberger \& Gilbert 1987). Chez B. plicatilis, polyphage consommant toute particule organique de taille convenable, en particulier du nanophytoplancton (Pourriot 1977), une faible vitesse de nage pourrait suffire pour assurer une alimentation minimale, donc une valeur de $S_{0}$ basse, dans un milieu aux ressources limitées mais constituées d'éléments de petite taille aisément ingérés.

Én ce qui concerne l'effet de la température, on observe chez $B$. calyciflorus que l'abaissement de la température entraîne une augmentation du seuil $S_{0}$ et une diminution du Ks (Tableau 3). En fait, ces variations ne font que traduire l'influence de la température sur la taille du corps puisqu'elles sont similaires à celles observées à la même température $\left(24^{\circ} \mathrm{C}\right)$ entre $B$. calyciflorus et $B$. angularis, de taille différente. Toutefois, les deux clones de $B$. calyciflorus ayant des caractères différents, l'existence d'une variation intraspécifique s'ajoutant à l'influence de la température ne peut être exclue.

\section{Remerciements}

Les auteurs remercient deux stagiaires, Catherine Bousquet et Abilio Lopez Neto, pour leur participation technique à l'expérimentation ainsi que Gerard Lacroix pour la lecture du manuscrit et ses suggestions. 


\section{Travaux cités}

Arfi R., Pagano M. \& Saint-Jean L. 1987. - Communautés zooplanctoniques dans une lagune tropicale (La lagune Ebrié, Côte d'Ivoire). Rev. Hydrobiol. trop., 20:21-35.

Birch L.C. 1948. - The intrinsic rate of natural increase of an insect population. J. Animal Ecol., 17 : 15-26.

Bottrell H.H., Duncan A., Gliwicz Z.M., Grygierek E., Herzig A., Hillbricht-Ilkowska A., Kurasawa A., Larsson P. \& Weglenska T. 1976. - A review of some problems in zooplankton production studies. Norw. J. Zool., 24 : 419-456.

Crowley P.H. 1975. - Natural selection and the Michaelis constant. J. theor. Biol., $50: 461-475$.

DeMott W.R. 1989. - The role of competition in zooplankton succession. In Plankton Ecology, Succession in Plankton Communities, U. Sommer Ed., Springer Verlag, Berlin : 195-252.

Duncan A. 1989. - Food limitation and body size in the life cycles of planktonic rotifers and cladocerans. Hydrobiologia, 186/187 : 11-28.

Gaudy R., Verriopoulosz G. \& Cervetto G. 1995. - Space and time distribution of zooplankton in a Mediterranean lagoon (Etang de Berre). Hydrobiologia, 300/301 :219-236.

Gliwicz Z. 1990. - Food thresholds and body size in cladocerans. Nature $3436259: 638-640$.

Halbach U. \& Halbach-Keup G. 1974. - Quantitative Beziehungen zwischen Phytoplankton und der Populationsdynamik des Rotators Brachionus calyciflorus Pallas. Befunde aus Laboratoriumsexperimenten und Freilanduntersuchungen. Arch. Hydrobiol., 73, 273-309.

Hiramaya K., Watanabe K. \& Kusano T. 1973. - Fundamental studies on physiology of Rotifer for its mass cultur - III. Influence of phytoplankton density and population growth. Bull. jap. Soc. sci. Fish., 39, 1123-1127.

Hiramaya K., Takagi K. \& Kimura H. 1979. - Nutritional effect of eight species of marine phytoplankton on population growth of the Rotifer, Brachionus plicatilis. Bull. jap. Soc. sci. Fish., 45, 1116.

Kerfoot W.C. \& Sih A. ed. 1987. - Predation, direct and indirect impacts on aquatic communities. Univ. Press New England, Hanover: $386 \mathrm{p}$.

Lazzaro X. \& Lacroix G. 1995. - Impact des poissons sur les communautés aquatiques. In Limnologie générale, Pourriot R. \& Meybeck M. Eds, Masson, Paris : 648-686.

Mourelatos S., Pourriot R. \& Rougier C. 1990. - Taux de filtration du rotifère Brachionus calyciflorus : comparaison des méthodes de mesure; influence de l'âge. Vie et Milieu, $40: 39-43$.

Pejler B. 1995. - Relation to habitat in Rotifers. Hydrobiologia, 313/314:267-278.

Pourriot R. 1965. - Recherches sur l'écologie des Rotiferes. Vie et Milieu, Suppl. 21, 224 p.

Pourriot R. 1980. - Workshop on culture techniques of rotifers. $H y$ drobiologia, $73:$ 33-35.
Pourriot R. 1995. - Réponses adaptatives du zooplancton à la prédation. In Limnologie générale, Pourriot R. \& Meybeck M. Eds Masson, Paris : 610-627.

Reynolds C.S. 1984. - The ecology of freshwater phytoplankton. Cambridge Univ. Press : $384 \mathrm{p}$.

Rocha O. \& Duncan A. 1985. The relationship between cell carbon and cell volume in freshwater algal species used in zooplankton studies. J. Plankton Res., $7: 279-294$.

Rothhaupt K.O. 1990. - Resource competition of herbivorous zooplankton : a review of approaches and perspectives. Arch. Hydrobiol. 118, 1-29.

Rothhaupt K.O. 1990a. - Differences in particle size-dependent feeding efficiencies of closely related rotifer species. Limnol. Oceanogr. $35: 16-23$.

Rothhaupt K.O. 1990b. - Population growth rates of two closely related Rotifer species : effects of food quantity, particle size, and nutritional quality. Freshw. Biol., 23, 561-570.

Ruttner-Kolisko A. 1972. - Der Einfluss von Temperature und Salzgehalt des Mediums auf Stoffwechsel- und Vermehrungsintensität von Brachionus plicatilis (Rotatoria). Verhand. deutsch. Zool. Ges., 65, 89-94.

Ruttner-Kolisko A. 1977. - Suggestions for the biomass calculation of plankton rotifers. Arch. Hydrobiol., Beih., Ergbn. Limnol. $8: 71-76$.

Schmid-Araya J.M. 1991. - The effect of food concentration on the life histories of Brachionus plicatilis (O.F.M.) and Encentrum linnhei Scott. Arch. Hydrobiol., 121, 87-102.

Segers H. 1995. - Nomenclatural consequences of recent studies on Brachionus plicatilis (Rotifera, Brachionidae). Hydrobiologia, 313/314: 121-122.

Snell T.W. \& Boyer E.M. 1988. - Thresholds for mictic female production in the rotifer Brachionus plicatilis (Muller). J. exp. mar. Biol. Ecol., 124, 73-85.

Snell T.W. \& Carrillo K. 1984. - Body size variation among strains of the rotifer Brachionus plicatilis. Aquaculture, 37 : 359-367.

Snell T.W. \& Hawkinson C.A. 1983. - Behavioral reproductive isolation among populations of the rotifer Brachionus plicatilis. Evolution, 37 : 1294-1305.

Stemberger R.S. \& Gilbert J.J. 1985. - Body size, food concentration, and population growth in planktonic rotifers. Ecology, 66 : 1151-1159.

Stemberger R.S. \& Gilbert J.J. 1987. - Rotifer threshold food concentrations and the size-efficiency hypothesis. Ecology, 68 : 181-187.

Vadstein O., Oie G. \& Olsen Y. 1993. - Particle size dependent feeding by the rotifer Brachionus plicatilis. Hydrobiologia, 255/256 : 261-267.

Walz N. 1987. - Comparative population dynamics of the rotifers Brachionus angularis and Keratella cochlearis. Hydrobiologia, 147, 209-213. 